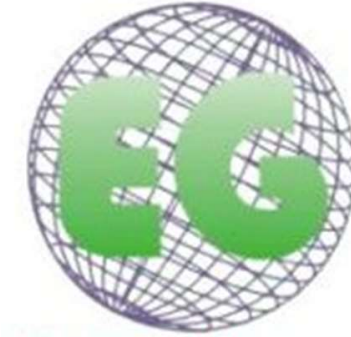

ISSN 1695-6141 N'57

\title{
Calidad de vida en adultos institucionalizados con parálisis cerebral
} infantil

Quality of life in institutionalized adults with cerebral palsy

\section{Laura Martínez-Traver ${ }^{1}$}

Águeda Cervera Gasch ${ }^{2}$

\begin{abstract}
${ }^{1}$ Fundación ASPROPACE, Centro de día y residencia ASPROPACE. Castellón de la Plana. España. ${ }^{2}$ Departamento de Enfermería.Facultad de Ciencias de la Salud.Universidad Jaume I. Castellón de la Plana. España. cerveraa@uji.es
\end{abstract}

\section{http://dx.doi.org/10.6018/eglobal.19.1.349901}

Recibido: 20/11/2018

Aceptado: 15/02/2019

\section{RESUMEN:}

Introduccion: La parálisis cerebral es considerada como un grupo de trastornos permanentes del desarrollo que originan limitaciones en la actividad diaria. Una de las complicaciones en las personas con parálisis cerebral es la disminución de la calidad de vida.

Objetivo: El objetivo de este estudio es conocer la calidad de vida de las personas adultas institucionalizadas con parálisis cerebral infantil y conocer si existen diferencias en el índice de calidad de vida en función de las características funcionales.

Método: Estudio observacional descriptivo trasversal en personas adultas institucionalizas con parálisis cerebral infantil realizado en los centros APCA y ASPROPACE entre marzo y septiembre de 2017. Se administró el cuestionario San Martín para medir la Calidad de Vida, Gross motor classification system, cuestionario Barthel, Escala CFCS para medir función motora, dependencia física, nivel de comunicación y relacionarlas con variables sociodemográficas y clínicas. La selección de los pariticipantes se llevó a cabo mediante un muestreo estratificado aleatorio. Se contó con la aprobación de las direcciones de los centros.

Resultados: Participaron 39 personas con edad media de 32,54 años. La puntuación media global del cuestionario San Martin fue de 102,97 puntos. Las dimensiones mas afectadas fueron bienestar material, desarrollo personal e incluison social. No se obtuvo significación estadística en la puntuación global del cuestionario en función de las variables secundarias.

Conclusiones: Los resultados de este estudio han demostrado que la Calidad de vida de adultos con parálisis cerebral en los sujetos estudiados es buena.

Palabras clave: Parálisis cerebral, calidad de vida destreza motora, actividades cotidianas, comunicación.

\section{ABSTRACT:}

Introduction: Cerebral palsy is considered a group of permanent developmental disorders that cause limitations in daily activity. One of the complications in people with cerebral palsy is the decrease in quality of life.

Objective: The objective of this study is to know the quality of life for institutionalized adults with infantile cerebral palsy and to know if there are differences in the quality of life index based on functional characteristics. 
Method: Cross-sectional descriptive observational study in institutionalized adults with infantile cerebral palsy performed in the APCA and ASPROPACE centers between March and September 2017. The San Martín questionnaire was administered to measure the Quality of Life, Gross motor classification system, Barthel questionnaire, Scale CFCS to measure motor function, physical dependence, level of communication and relate them with sociodemographic and clinical variables. The selection of the participants was carried out by random stratified sampling. It was approved by the centers' addresses.

Results: Participants were 39 people with an average age of 32.54 years. The overall average score of the San Martin questionnaire was 102.97 points. The most affected dimensions were material wellbeing, personal development and social incluison. No statistical significance was obtained in the overall score of the questionnaire based on the secondary variables.

Conclusions: The results of this study have shown that the quality of life of adults with cerebral palsy in the subjects studied is good.

Key words: cerebral palsy, quality of life, motor skills, activities of daily living communication.

\section{INTRODUCCIÓN}

La parálisis cerebral infantil $(\mathrm{PCl})$ no es una enfermedad específica, más bien se prefiere considerar a la $\mathrm{PCl}$ como un término descriptivo para un grupo de trastornos motores de origen cerebral que se ubican dentro de las discapacidades del desarrollo(1). Actualmente, se describe como un grupo de trastornos permanentes del desarrollo del movimiento y de la postura que originan limitaciones en la actividad y que son atribuibles a alteraciones no progresivas ocurridas en el desarrollo cerebral del feto ${ }^{(2)}$.

No existen criterios únicos para definir la calidad de vida $(\mathrm{CV})$, pero puede llegarse a una definición global como el nivel percibido de bienestar derivado de la evaluación que realiza cada persona de elementos objetivos y subjetivos en distintas dimensiones de su vida (3). La Organización Mundial de la Salud (OMS) define la CV como "la percepción individual de la propia posición en la vida dentro del contexto del sistema cultural y de valores en que se vive y en relación con sus objetivos, esperanzas, normas y preocupaciones" (4).

Las personas con discapacidades significativas conforman un grupo heterogéneo que tiene en común distintos aspectos, como la dependencia de otras personas en la mayoría de actividades de su vida diaria. A la hora de comunicarse hacen muy difícil utilizar escalas de calidad de vida autoinformadas, por lo tanto, la medición de la CV se realiza mayoritariamente mediante informes de otras personas que conocen bien a la persona con discapacidad significativa ${ }^{(5)}$.

A pesar de tratarse de una lesión no progresiva los síntomas sí que permanecen en la etapa adulta por lo que existen consecuencias sobre las funciones y actividades durante el crecimiento ${ }^{(6)}$, de hecho, entre cinco y quince años tras haber alcanzado la adolescencia o edad adulta temprana hay evidencia de una notable disminución en la movilidad que conlleva a una disminución de la capacidad funcional ${ }^{(7)}$ y la producción de cambios que tienen impacto sobre la calidad de vida (CV) de los pacientes ${ }^{(2)}$. Este impacto se refiere a la disminución de la CV debido a problemas físicos, patologías del habla y problemas de movilidad relacionados con actividades de la vida diaria (8).

Por tanto, los ojetivos de este estudio son: Conocer la calidad de vida de las personas adultas con parálisis cerebral infantil, mediante la Escala San Martín, y su afectación en el nivel de dependencia, nivel de comunicación y función motora asi como determinar qué aspectos hay que fomentar para obtener una mayor calidad de vida en adultos con parálisis cerebral infantil. 


\section{MÉTODO}

Se trata de un estudio observacional descriptivo transversal basado en el análisis de la calidad de vida mediante la Escala San Martín.

La población objeto de estudio fueron adultos con $\mathrm{PCl}$ de los centros de día y residencias de las fundaciones APCA y ASPROPACE de la Comunidad Valenciana. El estudio se realizó entre Marzo y Septiembre del 2017.

La selección de los pariticipantes se llevó a cabo mediante un muestreo estratificado aleatorio donde los estratos se correspondieron con a cada uno de los centros. No entraron a formar parte del estudio aquellas personas que no aceptaron ser incluidas en el estudio y que presentaron ingresos intermitentes tanto en centro de día como en residencia.

El cálculo del tamaño muestral se realizó en base al número total de pacientes de los centros participantes que cumplían los criterios de inclusión ( $N=59)$. De acuerdo con los resultados del programa GRANMO, se consideró suficiente una muestra aleatoria de 39 sujetos, considerando una confianza del 95\%, una precisión de +/-3 puntos y una desviación estándar de 15 puntos sobre la puntuación global del cuestionario escala San Martín. Se consideró un porcentaje de reposición del $20 \%$.

Para medir la variable principal del estudio, la calidad de vida, se utilizó el cuestionario validado Escala San Martin ${ }^{(5)}$. Esta escala permite evaluar la calidad de vida de las personas con discapacidades significativas a través de un informante que conoce bien a la persona (al menos desde hace tres meses) y que tiene oportunidades de observala durante periodos prolongados de tiempo y en diversos contextos. La escala consta de 95 ítems enunciados en tercera persona cuyas dimensiones, número de ítems, rango de puntuación y resultados de validez se muestran en la tabla 1.

Tabla 1. Dimensiones y propiedad psicométricas del cuestionario.

\begin{tabular}{llll} 
Dimensión & $\mathbf{N}^{\circ}$ ítems & Rango de puntuación & Correlación media \\
\hline Autodeterminación & 12 & $2-17$ & 0,552 \\
Bienestar emocional & 12 & $2-15$ & 0,549 \\
Bienestar físico & 12 & $2-15$ & 0,444 \\
Bienestar material & 12 & $2-14$ & 0,515 \\
Derechos & 12 & $2-15$ & 0,448 \\
Desarrollo personal & 12 & $2-15$ & 0,640 \\
Inclusión social & 12 & $2-16$ & 0,544 \\
Relaciones interpersonales & 11 & $2-15$ & 0,591 \\
Global & 95 & $16-122$ & ----
\end{tabular}

Las variables secundarias estudiadas fueron: nivel de dependencia física, nivel de comunicación, function motora. Se incluyeron las variables sociodemográficas y clínicas: Sexo (hombre/mujer), tipo parálisis cerebral (espástica / atáxica / discinética /mixta), tipo usuario (centro de día / residencia), provincia: (Castellón/Alicante) y edad en años cumplidos, siguiendo la clasificación de Martin, J.F. Los sujetos se clasificaron en adultos jóvenes 20-39 años / adultos medios 40-49 años / adultos maduros desde los 50 años ${ }^{(9)}$.

La dependencia física fue medida con el índice de Barthel ${ }^{(10)}$, test con una fiabilidad interobservador con índices de Kappa entre 0.47 y 1.00 , una fiabilidad intraobservador con un índice de kappa entre 0.84 y 0.97 . Este índice tiene diez dominios los cuales se valoran de forma diferente, pudiendo asignar $0,5,10$ o 15 puntos. La puntuación 
total de la escala puede variar entre 0 (completamente dependiente) y 100 (completamente independiente).

La habilidad de comunicación se midió con el Sistema de Clasificación de Comunicación Funcional (CFCS), que fue diseñado para evaluar la habilidad, de personas con parálisis cerebral, de comunicarse en diferentes ambientes (11). Esta escala debe ser realizada por el profesional que esté familiarizado con la comunicación de la persona seleccionando el nivel de rendimiento de la comunicación. La escala clasifica en 5 niveles.

La función motora fue medida por los equipos de enfermería mediante la escala Gross Motor Function Classification System (GMFCS) la cual consta de cinco niveles ${ }^{(12)}$. En un principio fue creada para la población infantil, pero con el paso del tiempo ha demostrado ser válida para clarificar también a los adultos. En su estudio de validación se obtuvo un coeficiente de kappa de 0.75 . La escala categoriza a la persona en 5 niveles, el nivel 1 representa mayor independencia en cuanto a la función motora gruesa y el nivel 5 el de menor independencia.

Todas estas escalas fueron administradas por el personal de enfermería.

Se realizó un análisis descriptivo de todas las variables en función de la naturaleza de las mismas. Para las variables categóricas se realizó una distribución de frecuencias y proporciones. Por otra parte, el análisis para las variables continuas se realizó mediante el cálculo de la media, mediana, desviación típica y máximo y mínimo para la media de cada una de las variables y dimensiones.

Se realizó un análisis bivariante para determinar si existían diferencias en las medias del Índice de Calidad de vida y sus dimensiones en función de las variables secundarias. Para la variable principal (ICV) y sus dimensiones se realizó un estudio de la normalidad mediante el test de Kolmogorov-Smirnov. En los resultados donde no se obtuvo una distribución normal de la variable se aplicaron los test no paramétricos (Test de Wilcoxon para la comparación de dos medias y el test de Kruskal Wallis para la comparación de más de tres medias). En las variables en las que se obtuvo una distribución normal se realizó el test de Levenne, para comprobar la igualdad o no de varianzas, en función de la variable secundaria a estudiar. Según los resultados se aplicó la prueba test-t para muestras independientes o el test ANOVA para la comparación de tres o más grupos, suponiendo varianzas iguales o no, en función de los resultados obtenidos por el test deLevenne.

Se utilizó el programa estadístico $\mathrm{R}$ commander, se estableció de significación estadística de $p<0,05$.

El proyecto se diseñó de acuerdo con la Ley Orgánica 3/2018, de 5 de diciembre (13) de Protección de Datos de Carácter Personal y se contemplaron las consideraciones éticas de la declaración de Helsinki.

Se respetaron los principios éticos de la investigación científica biomédica de acuerdo a la legislación española en materia de protección de datos. El cuestionario no recogía datos de carácter personal. Para garantizar la confidencialidad de los datos, toda la información se mantuvo codificada bajo contraseña. El proyecto cuenta con la autorización de la gerencia de los centros APCA y ASPROPACE. 


\section{RESULTADOS}

La muestra incluyó a 39 participantes (24 varones y 15 mujeres) con edades comprendidas entre los 20 y 56 años con una media de edad de 32,54 años $(\mathrm{DE}=8,61)$. Del total de los participantes 16 pertenecieron a centro de día y 23 a residencia y en cuanto a la provincia 14 pertenecían a la de Castellón y 25 a la de Alicante. En cuando al resto de variables secundarias la media de la muestra obtenida para el índice de Barthel fue de 16,41 (DE: $\pm 20,83$; IC del 95\%: 0-70) puntos y una mayor frecuencia $(n=28)$ en el grupo dependencia total. En el nivel de función motora y nivel de comunicación se obtuvieron mayores frecuencias en los niveles $5(n=27)$ y nivel $3(n=11)$ respectivamente (tabla 2$)$.

Tabla 2. Variables sociodemográficas y clínicas.

\begin{tabular}{|c|c|c|}
\hline Variable & Frecuencia & Porcentaje \\
\hline Sexo & & \\
\hline Hombre & 24 & 61,54 \\
\hline Mujer & 15 & 38,46 \\
\hline \multicolumn{3}{|l|}{ Tipo parálisis } \\
\hline Espástica & 2 & 5,13 \\
\hline Atáxica & 0 & \\
\hline Discinética & 0 & \\
\hline Mixta & 3 & 7,69 \\
\hline No especificada & 34 & 87,18 \\
\hline \multicolumn{3}{|l|}{ Tipo usuario } \\
\hline Centro de día & 16 & 41,03 \\
\hline Residencia & 23 & 58,97 \\
\hline \multicolumn{3}{|l|}{ Provincia } \\
\hline Castellón & 14 & 35,9 \\
\hline Alicante & 25 & 64,1 \\
\hline \multicolumn{3}{|l|}{ Edad (agrupada) } \\
\hline Adulto joven & 31 & 79,49 \\
\hline Adulto medio & 2 & 15,38 \\
\hline Adulto maduro & 6 & 5,13 \\
\hline \multicolumn{3}{|l|}{ Barthel } \\
\hline Dependencia total & 28 & 71,79 \\
\hline Dependencia severa & 9 & 23,08 \\
\hline Dependencia moderada & 2 & 5,13 \\
\hline \multicolumn{3}{|c|}{ Función motora } \\
\hline Nivel 1 & 1 & 2,56 \\
\hline Nivel 2 & 1 & 2,56 \\
\hline Nivel 3 & 4 & 10,26 \\
\hline Nivel 4 & 6 & 15,38 \\
\hline Nivel 5 & 27 & 69,23 \\
\hline \multicolumn{3}{|l|}{ Nivel de comunicación } \\
\hline Nivel 1 & 3 & 7,69 \\
\hline Nivel 2 & 7 & 17,95 \\
\hline Nivel 3 & 11 & 28,21 \\
\hline Nivel 4 & 8 & 20,51 \\
\hline Nivel 5 & 10 & 25,64 \\
\hline
\end{tabular}

La puntuación media global del cuestionario fue de 102,97 (DE \pm 9,07; IC del 95\%: 85120) puntos. Las dimensiones peor puntuadas fueron: bienestar material con una media de 9,53 (DE: $\pm 2,22$; IC del 95\%: 4-14) puntos, desarrollo personal 9,79 (DE: \pm 
2,39; IC del 95\%: 5-15) e inclusion social 9,79 (DE: $\pm 2,51$; IC del 95\%: 5-18). La tabla 3 ofrece el análisis de cada dimensión.

Tabla 3. Análisis descriptivo de los resultados de aplicación del cuestionario.

\begin{tabular}{llllll} 
& N & Media & SD & Mín & Máx. \\
\hline INDICE CALIDAD DE VIDA & 39 & 102,97 & 9,07 & 85 & 120 \\
\hline Autodeterminación & 39 & 11,58 & 2,73 & 6 & 15 \\
Bienestar emocional & 39 & 10,84 & 1,78 & 6 & 15 \\
Bienestar físico & 39 & 11,05 & 1,94 & 8 & 15 \\
Bienestar material & 39 & 9,53 & 2,22 & 4 & 14 \\
Derechos & 39 & 11,03 & 2,06 & 6 & 15 \\
Desarrollo personal & 39 & 9,79 & 2,39 & 5 & 15 \\
Inclusión social & 39 & 9,79 & 2,51 & 5 & 18 \\
Relaciones interpersonales & 39 & 10,51 & 2,21 & 6 & 15 \\
$\quad$ & & & & & \\
Lectura: N= Número de encuestado & & & & & \\
$\quad$ DE= Desviacion estandar & & & & & \\
$\quad \begin{array}{lllll}\text { Min= Valor minimo de la respuesta } \\
\text { Max= Valor máximo de la respuesta }\end{array}$ & & & & &
\end{tabular}

La tabla 4 recoge la comparación de medias del global del cuestionario y de cada una de las dimensiones en función de las variables secundarias.

Tabla 4. Significacionestadistica obtenida en la realización de las pruebas estadísticas del ICV y sus dimensiones en función de las variables secundarias.

\begin{tabular}{|c|c|c|c|c|c|c|c|c|c|}
\hline Variable & ICV & $\begin{array}{l}\text { Autodeter } \\
\text { minación }\end{array}$ & $\begin{array}{l}\text { Bienestar } \\
\text { Emocional }\end{array}$ & $\begin{array}{l}\text { Bienes } \\
\text { tar } \\
\text { Físico }\end{array}$ & $\begin{array}{l}\text { Bienes } \\
\text { tar } \\
\text { Material }\end{array}$ & $\begin{array}{l}\text { Dere } \\
\text { chos }\end{array}$ & $\begin{array}{l}\text { Desarro } \\
\text { Ilo } \\
\text { personal }\end{array}$ & $\begin{array}{l}\text { Inclusión } \\
\text { social }\end{array}$ & $\begin{array}{l}\text { Relacio } \\
\text { nes } \\
\text { interper } \\
\text { sonales }\end{array}$ \\
\hline Sexo & $0.345^{1}$ & $0.114^{1}$ & $0.466^{3}$ & $0.872^{3}$ & $0.893^{1}$ & $0.755^{3}$ & $0.678^{1}$ & $0.403^{3}$ & $0.340^{3}$ \\
\hline $\begin{array}{l}\text { Tipo } \\
\text { parálisis }\end{array}$ & $0.401^{2}$ & $0.954^{2}$ & $0.384^{4}$ & $0.384^{4}$ & $0.328^{2}$ & $0.384^{4}$ & $0.775^{2}$ & $0.644^{4}$ & $0.644^{4}$ \\
\hline & $0.539^{1}$ & $0.593^{1}$ & $0.325^{3}$ & $0.284^{3}$ & $0.437^{1}$ & $0.311^{3}$ & $0.717^{1}$ & $0.212^{3}$ & $0.771^{3}$ \\
\hline Provincia & $0.069^{5}$ & $0.070^{1}$ & $0.416^{3}$ & $0.025^{3}$ & $0.036^{5}$ & $0.071^{3}$ & $0.224^{5}$ & $0.030^{3}$ & $0.087^{3}$ \\
\hline $\begin{array}{l}\text { Grupo } \\
\text { edad }\end{array}$ & $0.376^{2}$ & $0.747^{2}$ & $0.402^{4}$ & $0.402^{4}$ & $0.608^{6}$ & $0.402^{4}$ & $0.061^{2}$ & $0,676^{4}$ & $0.676^{4}$ \\
\hline $\begin{array}{l}\text { Grupo } \\
\text { Barthel }\end{array}$ & $0.698^{2}$ & $0.626^{2}$ & $0.712^{4}$ & $0.712^{4}$ & $0.945^{2}$ & $0.712^{4}$ & $0.115^{2}$ & $0,139^{4}$ & $0.435^{4}$ \\
\hline $\begin{array}{l}\text { Nivel } \\
\text { función } \\
\text { motora }\end{array}$ & $0.586^{2}$ & $0.574^{2}$ & $0.839^{4}$ & $0.999^{4}$ & $0.882^{2}$ & $0.274^{4}$ & $0.368^{2}$ & $0,163^{4}$ & $0.204^{4}$ \\
\hline $\begin{array}{l}\text { Nivel de } \\
\text { comunica } \\
\text { ción }\end{array}$ & $0.051^{2}$ & $0.007^{2}$ & $0.181^{4}$ & $0.181^{4}$ & $0.153^{2}$ & $0.181^{4}$ & $0.059^{2}$ & $0,241^{4}$ & $0.241^{4}$ \\
\hline
\end{tabular}

Leyenda:

${ }^{1}$ Prueba test-t para muestras independientes suponiendo igualdad de varianzas

${ }_{3}^{2}$ Test ANOVA suponiendo igualdad de varianzas

${ }^{3}$ Test de Wilcoxon para dos muestras

${ }_{5}^{4}$ Test de Kruskal-wallis

${ }^{5}$ Prueba test-t para muestras independientes no suponiendo igualdad de varianzas

${ }^{6}$ Test ANOVA no suponiendo igualdad de varianzas 
No se encontró significación estadística para afirmar que existen diferencias en el nivel medio del global del cuestionario en función de las variables secundarias. En cuanto al análisis de cada una de las dimensiones del cuestionario en función de las variables secundarias no se encontró significación estadística para afirmar que existen diferencias en el nivel medio del ICV y sus dimensiones en función del nivel de dependencia física y de la función motora. En el análisis del nivel de comunicación sí se encontró evidencia estadística que nos confirmó la existencia de diferencias en el nivel medio de la autodeterminación en función de esta variable ( $p$ valor $=0,00794$ ) al realizar la comparación de medias mediante el test ANOVA. Una comparación de medias dos a dos nos permitió observar que las diferencias en la media de la autodeterminación se encontraron entre los niveles 1-2-3 o con respecto a los niveles 4-5. Tambien se observó significación estadística para afirmar que existían diferencias en la media del bienestar físico en función de la provincia ( $p$ valor=0.025). Al realizar un contraste de hipótesis unilateral se observó que la media obtenida en bienestar físico en la provincia de Alicante era menor que la media obtenida en la provincia de Castellón. En esta variable también se observaron diferencias en relación a la inclusión social en función de la provincia ( $p$ valor=0.030). En este aspecto también se realizó un contraste de hipótesis unilateral y se obtuvo que la media obtenida en inclusión social en la provincia de Alicante era mayor que la media obtenida en la provincia de Castellón.

\section{DISCUSIÓN}

Los resultados obtenidos se encuentran en consonancia con los obtenidos por M. Badia- Corbella et al., ${ }^{(4)}$ que concluyeron que la calidad de vida de los pacientes con parálisis cerebral en proceso de envejecimiento, en general, es buena. En cuando a la comparación en función del sexo esto coincide con los resultados obtenidos en estudio llevado a cabo por Ross et al., ${ }^{(14)}$ donde al comparar la calidad de vida en adultos con parálisis cerebral no se encontraron diferencias estadísticas significativas entre hombres y mujeres.

En cuanto al nivel de CV y la dependencia física, no se han encontrado diferencias estadísticamente significativas en el ICV y sus dimensiones en función del nivel de Barthel obtenido y el nivel de función motora. En este trabajo se ha demostrado que la función motora según la escala GMFCS no obtiene diferentes puntuaciones, en cuanto al ICV y las dimensiones de la calidad de vida.

Esto entra en contraposición con el estudio llevado a cabo por Braccialli et al., ${ }^{(15)} \mathrm{en}$ el que se señala que la gravedad de la discapacidad motora sí afectan a la calidad de vida de las personas con PC. Por otro lado coincide con otro estudio donde no encontraron evidencias estadísticas de que la función motora afecte al ICV en una muestra de niños con parálisis cerebral ${ }^{(16)}$

También se han encontrado diferencias estadísticamente significativas en la calidad de vida en función del nivel de comunicación según la escala CFCS donde podemos afirmar que el nivel de comunicación de la escala CFCS afecta a la dimensión de autodeterminación ya que para las personas con niveles de comunicación I y II se ha demostrado que hay evidencia estadística suficiente para afirmar que tienen mejores resultados en autodeterminación que las personas con un nivel de comunicación III, IV y $\mathrm{V}$ según la escala de comunicación CFCS. Autores como M. Hickey y P. Moore concluyeron que una de las dimensiones de la CV más afectadas en personas 
adolescentes con parálisis cerebral era la inclusión social(17) .De la misma forma el estudio llevado a cabo por A. Colver, M. Rap et al. (18), también determinó que las dimensiones más afectadas en adolescentes con parálisis cerebral eran la autodeterminación y la inclusión social. Ambos resultados entran en concordancia con los obtenidos en este estudio.

Las dimensiones de la calidad de vida en las que se han obtenido puntuaciones medias más bajas han sido: desarrollo personal, inclusión social y bienestar material. En un estudio llevado a cabo por Park et al.,(19) también se encontraron medias inferiores en inclusión social pero en este caso la otra dimensión con una media inferior al resto fue la dimensión de relaciones interpersonales.

Al ser un estudio transversal encontramos limitaciones con la obtención de las muestras al encontrar negación por parte de los centros en la participación en el estudio además la gran dispersión geográfica requiere grandes desplazamientos lo que dificulta el seguimiento exhaustivo en la recogida de datos. También debemos considerar los resultados con cautela ya que las frecuencias para algunas de las variables secundarias resultaron ser pequeñas con valores menores a 5 personas.

\section{CONCLUSIÓN}

Los resultados de este estudio han demostrado que la Calidad de vida de adultos con parálisis cerebral, pertenecientes a los centros APCA Y ASPROPACE es, en general, buena.

Existen diferencias en la dimensión bienestar físico y en la dimensión de inclusión social entre provincias y la dimensión de autodeterminación se ve afectada en función del nivel de comunicación. La edad media muestra que estamos frente a una población jóven. Ha surgido la necesidad de mejorar la comunicación en las personas con niveles más elevados en la escala CFCS para intentar aumentar los niveles de autodeterminación. Además se ha observado que en los centros de Castellón se debe centrar cierta parte del trabajo en aumentar la inclusión social y en los centros de las provincias de Alicante en trabajar la dimensión del bienestar emocional.

\section{REFERENCIAS}

1. Gulati S, Sondhi V. Cerebral Palsy: An Overview. Indian J Pediatr. 2018 Nov;85(11):1006-1016. doi: 10.1007/s12098-017-2475-1. Disponible en: https://link.springer.com/article/10.1007\%2Fs12098-017-2475-1

2. Wimalasundera N, Stevenson VL. Cerebral palsy. Pract Neurol. 2016 Jun;16(3):18494. doi: 10.1136/practneurol-2015-001184. Disponible en: https://pn.bmj.com/content/16/3/184.long

3. Colver A, Rapp M, Eisemann N, Ehlinger V, Thyen U, Dickinson HO, Parkes J, Parkinson K, Nystrand M, Fauconnier J, Marcelli M, Michelsen SI, Arnaud C. Selfreported quality of life of adolescents with cerebral palsy: a cross-sectional and longitudinal analysis. Lancet. 2015 Feb 21;385(9969):705-16. doi: 10.1016/S01406736(14)61229-0.

Disponible en https://www.sciencedirect.com/science/article/pii/S0140673614612290?via\%3Dihub

4. Badía Corbella, M., Carrasco Trenado, J., Orgaz Baz, M. B., \& Escalonilla García, J. M. (2018). Calidad de vida percibida por personas adultas con discapacidades del desarrollo versus la informada por profesionales Siglo Cero Rev Española sobre 
Discapac Intelect. 2016;47(1):7. Disponible en: http://revistas.usal.es/index.php/02101696/article/view/scero20161721

5. Verdugo MA, Gómez LE, Arias B, Navas P, Schalock RL. Measuring quality of life in people with intellectual and multiple disabilities: validation of the San Martín scale. Res Dev Disabil. 2014 Jan;35(1):75-86. doi:10.1016/j.ridd.2013.10.025. Disponible en: https://www.sciencedirect.com/science/article/pii/ S0891422213004691?via\%3Dihub

6. Fabricio Espinoza Ortiz. Aproximacion Teorica Al Concepto De Calidad De Vida.2014;No14,2014:331-47. Disponible en: https://revistaselectronicas.ujaen.es/ index.php/rae/article/view/1801/1559

7. Jiang B, Walstab J, Reid SM, Davis E, Reddihough D. Quality of life in Young adults with cerebral palsy. Disabil Health J. 2016 Oct;9(4):673-81. doi: 10.1016/j.dhjo.2016.04.006. Disponible en: https://www.sciencedirect.com/ science/article/pii/S1936657416300450?via\%3Dihub

8. Scherer R, Billinger M, Wagner J, Schwarz A, Hettich DT, Bolinger E, Lloria Garcia M, Navarro J, Müller-Putz G. Thought-based row-column scanning communication board for individuals with cerebral palsy. Ann Phys Rehabil Med. 2015 Feb;58(1):14-22. doi: 10.1016/j.rehab.2014.11.005. Disponible en: https://www.sciencedirect.com/science/article/pii/S1877065714018430?via\%3Dihub

9. Martin J-F. Los factores definitorios de los grandes grupos de edad de la población: tipos, subgrupos y umbrales. Geo Crítica / Scripta Nova. Revista electrónica de geografía y ciencias sociales. Barcelona: Universidad de Barcelona. p. vol. IX, núm. 190. Disponible en: http://www.ub.edu/geocrit/sn/sn-190.htm

10. Cid-Ruzafa J, Damián-Moreno J. Valoración de la discapacidad física: el indice de Barthel. Rev Esp Salud Publica. 1997;71(1):127-37. Available from: http://scielo.isciii.es/scielo.php?script=sci arttext\&pid=S1135-57271997000200004

11. Compagnone E, Maniglio J, Camposeo S, Vespino T, Losito L, De Rinaldis M, et al. Functional classifications for cerebral palsy: Correlations between the gross motor function classification system (GMFCS), the manual ability classification system (MACS) and the communication function classification system (CFCS). Res Dev Disabil. 2014;35(11):2651-7. Disponible en: http://dx.doi.org/10.1016/j.ridd.2014.07.005.

12. Andrea E, Mejía C, Cristina A, Ávila Q, Milena D, Vidal D. Escala Gross Motor Function Measure . Una revisión de la literatura. 2014;2(8):11-21. Disponible en: https://www.researchgate.net/publication/304662448 Escala Gross Motor Function Measure Una revision de la literatura

13. Ley Orgánica $3 / 2018$, de 5 de diciembre, de Proteccion de Dato Personales y garantia de los derechos digitales. Disponible en: https://boe.es/diario boe/txt.php?id=BOE-A2018-16673

14. Ross SM, MacDonald M, Bigouette JP. Effects of strength training on mobility in adults with cerebral palsy: A systematic review. Disabil Health J. 2016 Jul;9(3):375-84. doi: 10.1016/j.dhjo.2016.04.005.

Disponible en: https://www.sciencedirect.com/science/article/pii/S1936657416300449?via\%3Dihub

15. Braccialli, L. M., Almeida, V. S., Sankako, A. N., Silva, M. Z., Braccialli, A. C., Carvalho, S. M., \& Magalhães, A. T. (2016). Translation and validation of the Brazilian version of the Cerebral Palsy Quality of Life Questionnaire for Children-child report. Jornal de pediatria, 92(2), 143-148. Disponible en: https://www.sciencedirect.com/science/article/pii/S0021755715001722?via\%3Dihub

16. Pashmdarfard M, Amini M, Badv RS, Namazi NG, Rassafiani M. Does parent report gross motor function level of cerebral palsy children impact on the quality of life in these children? Iran J Child Neurol. 2017;11(4):52-7. Available from: https://www.ncbi.nlm.nih.gov/pubmed/29201124 
17. Hickey M, Moore P. Quality of life in adolescents with cerebral palsy. Lancet [Internet]. 2015;385(9969):670-2.

Available from:

http://dx.doi.org/10.1016/S01406736(14)61599-3

18. Colver, A., Rapp, M., Eisemann, N., Ehlinger, V., Thyen, U., Dickinson, H. O., ... \& Marcelli, M. (2015). Self-reported quality of life of adolescents with cerebral palsy: a cross-sectional and longitudinal analysis. The Lancet, 385(9969), 705-716. Disponible en:

https://www.sciencedirect.com/science/article/pii/S0140673614612290?via\%3Dihub

19. Park TS, Dobbs MB, Cho J. Evidence Supporting Selective Dorsal Rhizotomy for Treatment of Spastic Cerebral Palsy. Cureus. 2018 Oct 19;10(10):e3466. doi:10.7759/cureus.3466. Disponible en: https://www.ncbi.nlm.nih.gov/pmc/ articles/PMC6300384/ 